tain," says Cy Stein, professor of medicine and molecular pharmacology at the Albert Einstein College of Medicine in New York. By acting on ApoB, a carrier for lipids, mipomersen may also affect fat accumulation in the liver. Suppression of ApoB may lead to the accumulation of fats in cellular lipids, which might trigger the raised liver enzyme levels observed. This idea is supported by some patients with a genetic condition called hypobetalipoproteinemia, who cannot make ApoB and have low levels of LDL. Some of these patients accumulate liver fat. Thus, mipomersen could, in effect, be mimicking that condition, says Robert Hegele, a professor of medicine and biochemistry and director of the Blackburn Cardiovascular Genetics Laboratory at Robarts Research Institute in London, Ontario. Still, "It's all speculation [at this point]," he hastens to add.

Antisense therapies as a class, on the other hand, do have off-target effects of their own. One issue is that the highly charged phosphorothioate backbone binds tightly to charged residues in proteins-a property that helps them avoid elimination via the kidney through association with albumin proteins. But this property might also lead to the binding of antisense to proteins on the surface of hepatocytes, perhaps mimicking heparin and leading to abnormalities, according to Stein.

Despite the recent setback, Genzyme management, which is partnered with Isis on mipomersen, remains sanguine, noting that the effects on liver enzymes were reversible. "Physicians can manage (side effects) by backing off the medication because they're seeing such a significant drop in LDL," says Paula Soteropoulos, vice president and general manager of Genzyme's cardiovascular business. But some analysts think it's likely that mipomersen will be approved only for homozygous $\mathrm{FH}$ patients, who cannot metabolize LDL due to a lack of functional LDL receptors responsible for clearing LDL from plasma. Even if it were approved for a broader population, mipomersen might not have sufficient advantages to convince physicians to switch from small-molecule statin therapies that are administered orally rather than subcutaneously injected.

In any case, Genzyme plans to file in the first half of 2011 in the US and Europe, targeting patients with homozygous $\mathrm{FH}$ and possibly severe hypercholesterolemia. Both indications together represent $\sim 25,000$ patients in the US and Europe.
The impressive efficacy of mipomersen is testament to Isis's investment and optimization of second-generation antisense technology. These second-generation chemistries improve stability and binding, Soteropoulos says, and Isis has spun off or licensed the technology to other companies, such as OncoGeneX, located in Bothell, Washington (Table 1), and Altair Therapeutics, of San Diego.

Not everyone is convinced that a rejuvenation in antisense approaches is on the horizon, however. "Isis is the only company left. Other companies have converted to CpG or siRNA [small interfering RNA] approaches," says John Rossi, a professor of molecular and cellular biology at the City of Hope's Beckman Research Institute, Duarte, California. The main advantage of RNA interference (RNAi) over antisense has been its greater potency. "RNAi is long lasting. Once it's engaged the RNA silencing complex, [siRNA] can last for weeks," says Rossi. University of Pennsylvania researcher Gewirtz, agrees: "It just seems easier to find an RNA molecule that gets you into the game than it is to find an oligo. That's why RNAi became so widely accepted-it just works for everybody."

Even so, siRNA and CpG suffers from the same issues of off-target effects and delivery as antisense, and the latter has other advantages. Its easier to manufacture than siRNA, and because antisense has been around longer, there is more clinical experience behind it. There have been 20 or so clinical studies involving antisense, according to Tenthoff, whereas RNAi trials are still in the single digits. "I don't think antisense is yet giving way to RNAi. Antisense is still a more clinically experienced technology," says Tenthoff.

"I'm very enthusiastic about both approaches," says Raymond P. Warrell Jr., CEO of Genta. "The advantage of antisense is that there are now 15-plus years of clinical experience with it. The folks working primarily on RNAi are in the process of relearning a lot of [those] lessons." He expects the two technologies to ultimately be complementary. "Whether you use RNA or DNA depends to some extent on the target and to some extent the technique you have experience with. The challenge remains first and foremost to identify a critical target so you have a high level of confidence that knocking it out or down will have a transformative effect on the disease," says Warrell.

Jim Kling Bellingham, Washington
IN brief

\section{Orphans on the rise}

The number of drug approvals for orphan indications has doubled in recent years, according to a report from the Tufts Center for the Study of Drug Development. The independent, nonprofit research group at Tufts University in Boston, found that between 2000 and 2002 the US Food and Drug Administration (FDA) approved 208 orphan drugs, and the number climbed to 425 between 2006 and 2008. The increase could reflect the fact that orphan diseases are simple to target as they are often underpinned by a single genetic cause. But financial incentives for pursuing orphan drugs, such as a waiver of the FDA's $\$ 1.4$ million filing fee, long marketing exclusivity and high prices charged are likely factors, too. According to FDA data, biotech firms generate $50 \%$ of orphan drug applications and academia another $25 \%$. Pharma makes up less than $25 \%$ of the total probably because "orphan drugs do not regularly fit their business model," says Tim Coté, director of the FDA's Office of Orphan Product Development. That has been changing, however. Regeneron, of Tarrytown, New York, has seen this firsthand with Arcalyst (rilonacept), an interleukin-1 (IL-1) 'trap' to treat cryopyrinassociated periodic syndromes (CAPS), a rare disease that affects only a few thousand people globally. In 2003, Novartis of Basel terminated a collaboration with Regeneron over the IL-1 trap, because the Swiss pharma was not interested in the small market for CAPS. Later, Novartis developed an IL-1 antibody for CAPS, Ilaris (canakinumab), which gained approval in July 2009.

Nadine Kolas

\section{Pharma's Asian syndicate}

Three big pharmas-Pfizer, Merck, and Eli Lilly_are pooling their resources to set up an independent nonprofit company to spur research into innovative treatments for cancers common in Asian populations. The new Asian Cancer Research Group (ACRG) will build an open-access pharmacogenomic cancer database, which will be made publicly available to researchers in the field. Wu Jun, vice president of Xiangxue Pharmaceutical, Guangzhou, says, "It will save Western companies time and money and is good news for patients in China." The joint venture will focus initially on lung and gastric cancers and aims to gather 2,000 tissue samples over the next two years. "ACRG could get more data from Asia and spend less on research compared with what they spend in the West," says Wu. ACRG is an example of a growing trend in precompetitive collaborations. The same three companies have done it before with Enlight Biosciences (Nat. Biotechnol. 26, 960-961, 2008), an R\&D startup for developing drug discovery tools. The pharma giants are searching for ways to capture the emerging Asian markets. Plans for ACRG were already underway before last year's decision by the Chinese government to invest 850 billion yuan ( $\$ 125$ billion) on healthcare reform, according to a spokesperson for Merck. Bea Perks 